\title{
Endoscopic Suturing for the Prevention and Treatment of Complications Associated with Endoscopic Mucosal Resection of Large Duodenal Adenomas
}

\author{
Jaeil Chung, Kelly Wang, Alexander Podboy, Srinivas Gaddam, and Simon K. Lo \\ Karsh Division of Gastroenterology and Hepatology, Cedars-Sinai Medical Center, Los Angeles, CA, USA
}

Background/Aims: Endoscopic mucosal resection (EMR) is the primary treatment for duodenal adenomas; however, it is associated with a high risk of perforation and bleeding, especially with larger lesions. The goal of this study was to demonstrate the feasibility and safety of endoscopic suturing (ES) for the closure of mucosal defects after duodenal EMR.

Methods: Consecutive adult patients who underwent ES of large mucosal defects after EMR of large $(>2 \mathrm{~cm})$ duodenal adenomas were retrospectively enrolled. The OverStitch ES system was employed for closing mucosal defects after EMR. Clinical outcomes and complications, including delayed bleeding and perforation, were documented.

Results: During the study period, ES of mucosal defects was performed in seven patients in eight sessions (six for prophylaxis and two for the treatment of perforation). All ES sessions were technically successful. No early or delayed post-EMR bleeding was recorded. In addition, no clinically obvious duodenal stricture or recurrence was encountered on endoscopic follow-up evaluation, and no patients required subsequent surgical intervention.

Conclusions: ES for the prevention and treatment of duodenal perforation after EMR is technically feasible, safe, and effective. ES should be considered an option for preventing or treating perforations associated with EMR of large duodenal adenomas.

Clin Endosc 2022;55:95-100

Key Words: Duodenal adenoma; Endoscopic mucosal resection; Endoscopic suturing

\section{INTRODUCTION}

Duodenal adenomas are uncommon lesions with a reported prevalence of $0.3 \%$ to $4.6 \%$, often incidentally found in patients undergoing esophagogastroduodenoscopy. ${ }^{1-4}$ Approximately $40 \%$ of duodenal adenomas are sporadic, and the remaining $60 \%$ are found in patients with familial adenomatous polyposis. ${ }^{5,6}$ The standard treatment for a solitary duodenal

Received: October 21, 2020 Revised: November 21, 2020

Accepted: December 7, 2020

Correspondence: Simon K. Lo

Karsh Division of Gastroenterology and Hepatology, Cedars-Sinai Medical Center, 8700 Beverly Blvd., Los Angeles, CA 90048, USA

Tel: +1-310-423-6082, Fax: +1-310-423-1826, E-mail: Simon.Lo@cshs.org

ORCID: https://orcid.org/0000-0002-1263-0400

(cc) This is an Open Access article distributed under the terms of the Creative Commons Attribution Non-Commercial License (http://creativecommons.org/ licenses/by-nc/3.0) which permits unrestricted non-commercial use, distribution, and reproduction in any medium, provided the original work is properly cited. adenoma is endoscopic polypectomy, which includes endoscopic submucosal dissection (ESD) and endoscopic mucosal resection (EMR) ${ }^{7,8}$ However, advanced technical expertise is required to safely perform ESD, which has limited its widespread use. ${ }^{9}$ Indeed, the difficulty of ESD stems from the anatomical features of the duodenum, with increased vascularity in the submucosal layer and a thin muscularis propria, posing substantial risks of complications such as bleeding and perforation. ${ }^{10}$ Consequently, EMR is more commonly employed in Western countries. ${ }^{9,11}$

Although EMR of duodenal lesions can often achieve favorable en bloc resection rates, it is still associated with a high risk of perforation and bleeding, with potentially devastating consequences. ${ }^{11}$ Delayed perforation has been reported in $1 \%$ of non-ampullary adenomas treated with EMR; however, it could be significantly more frequent after the endoscopic resection of large-sized adenomas. ${ }^{12,13}$ Patients with perforation are typically referred for emergent surgery, although it may still result 
in significant mortality and morbidity. ${ }^{12,14,15}$ Closure of submucosal defects in the duodenum has been shown to decrease the number of delayed adverse events associated with submucosal dissection. ${ }^{16}$ The OverStitch endoscopic suturing (ES) system (Apollo Endosurgery, Austin, TX, USA), currently the only suturing device commercially available in the United States, is specifically designed for tissue approximation and allows the creation of either continuous running or interrupted stitches. ${ }^{17}$ The device has been shown to reliably appose tissues and to close perforations throughout the gastrointestinal tract. ${ }^{16}$ It has been employed for closing persistent gastrocutaneous fistula or active ulcers, and for fixing esophageal stents. ${ }^{18-20}$ However, the application of ES with the OverStitch system to prevent or treat duodenal perforations associated with duodenal EMR has not been well studied.

Thus, the aim of this study was to assess the technical feasibility, safety, and outcomes of ES for the closure of mucosal defects resulting from duodenal EMR.

\section{MATERIALS AND METHODS}

\section{Patient population}

This was a retrospective study of consecutive adult patients who underwent ES of large mucosal defects after EMR at Cedars-Sinai Medical Center over a 9-month period (March 2019-November 2019). Patients with benign duodenal mucosal adenomas of at least $20 \mathrm{~mm}$ in size, who were treated with EMR followed by attempted closure with ES, were considered eligible for inclusion in this study. The study was approved by the Institutional Review Board of Cedars-Sinai Medical Center (approval no. 00000346, Los Angeles, CA, USA).

\section{Endoscopic mucosal resection and suturing}

EMRs were performed by a single endoscopist (SKL), and the procedures were performed under monitored anesthesia care. After the submucosal injection of a mixture of epinephrine (dilution 1:10,000), methylene blue, and normal saline, EMR was performed using a single-use, braided snare (K001; Olympus America, Center Valley, PA, USA). Hot forceps (HDBF-2.4SN-230-S; Cook Medical, Winston-Salem, NC, USA) was used to ablate tissue that could not be captured with the EMR snare. A forward-viewing gastroscope, pediatric colonoscope, or side-viewing duodenoscope was used at the discretion of the endoscopist. After the completion of EMR, ES was performed with a double-channel endoscope (GIF 2T-180; Olympus America) preloaded with an OverStitch ES device, orally inserted to the location of the EMR. The postEMR mucosal defect was closed using interrupted stitches, with the primary intention of visibly closing the entire EMR resection defect. For each suture, the distal end of the mucosa was first captured and the proximal end was subsequently captured before cinching. Each suture was placed perpendicular to the duodenal folds to decrease the risk of duodenal luminal narrowing. Patients were discharged on the same day after the procedure, unless periprocedural complications occurred. All intraoperative and postprocedural clinical events were managed according to the standard of care and recorded, including those reported during a telephone visit performed on days 7-14.

\section{Data collection and analysis}

The patients' demographic and clinical data, including age, sex, pathologic diagnosis, lesion size, location, procedure outcomes, adverse events, and follow-up outcomes were reviewed. The collected data were stored in Microsoft Excel (Microsoft Co., Redmond, WA, USA). Data are reported as means and standard deviations for continuous variables, and as percentages for nominal variables. The primary study outcomes (delayed bleeding and perforation) were quantified.

\section{RESULTS}

During the study period, ES of mucosal defects was performed in seven patients in eight sessions (Table 1). Of the eight sessions, six were performed for prophylaxis and two for perforation (at 3 and 14 hours after EMR). All patients were referred from outside hospitals. The mean age of the patients was $72.7 \pm 11.7$ years. The patients in this case series included five women and two men, of whom six were Caucasian and one was Asian. Two patients underwent prior duodenal EMR procedures. All lesions were removed with EMR, and the mean lesion size was $35.7 \pm 15.7 \mathrm{~mm} \times 2.1 \pm 1.1 \mathrm{~mm}$. The location of the lesions included the duodenal bulb $(n=1)$, descending duodenum $(n=5)$, and horizontal duodenum (D2D3 junction; $n=1$ ). Histopathologic evaluation of the resected lesions revealed tubular adenoma (TA) with low-grade dysplasia $(n=6)$ and TA with high-grade dysplasia $(n=1)$.

All ES sessions were technically successful, although one stitch in a prophylactic case was intraprocedurally noted to be loose (Fig. 1). The location of the stitch was complicated by a delayed perforation; however, it was completely closed in another ES session without further adverse events or interventions (Fig. 1). The average number of stitches per case was $3.3 \pm 0.7$. In four prophylactic cases, clips were initially applied but were deemed unsuccessful or inadequate before the ES approach was employed (Fig. 2). Five patients with uncom- 
Table 1. Characteristics of the Study Patients

\begin{tabular}{ccccccccc}
\hline Patient & $\begin{array}{c}\text { Age } \\
\text { (years) }\end{array}$ & Sex & Location & Pathology & $\begin{array}{c}\text { Size } \\
(\mathbf{m m})\end{array}$ & $\begin{array}{c}\text { Involvement of } \\
\text { Papilla }\end{array}$ & $\begin{array}{c}\text { Hospital stay } \\
\text { (days) }\end{array}$ & $\begin{array}{c}\text { Complications } \\
\text { Surgery/ } \\
\text { death }\end{array}$ \\
\hline 1 & 80 & F & Horizontal & Tubular adenoma (LGD) & 50 & No & 10 & Delayed perforation \\
No \\
2 & 74 & F & Bulb & Tubular adenoma (LGD) & 25 & No & 1 & No \\
\hline 3 & 77 & M & Descending & Tubular adenoma (LGD) & 20 & Yes & 1 & Pancreatitis \\
4 & 77 & F & Descending & Tubular adenoma (LGD) & 35 & No & 0 & No \\
\hline 5 & 74 & M & Descending & Tubular adenoma (HGD) & 50 & Yes & 0 & No \\
\hline 6 & 61 & F & Descending & Tubular adenoma (LGD) & 45 & Yes & 1 & Delayed perforation \\
\hline 7 & 66 & F & Descending & Tubular adenoma (LGD) & 30 & No & 24 & No \\
\hline
\end{tabular}

HGD, high-grade dysplasia; LGD, low-grade dysplasia.
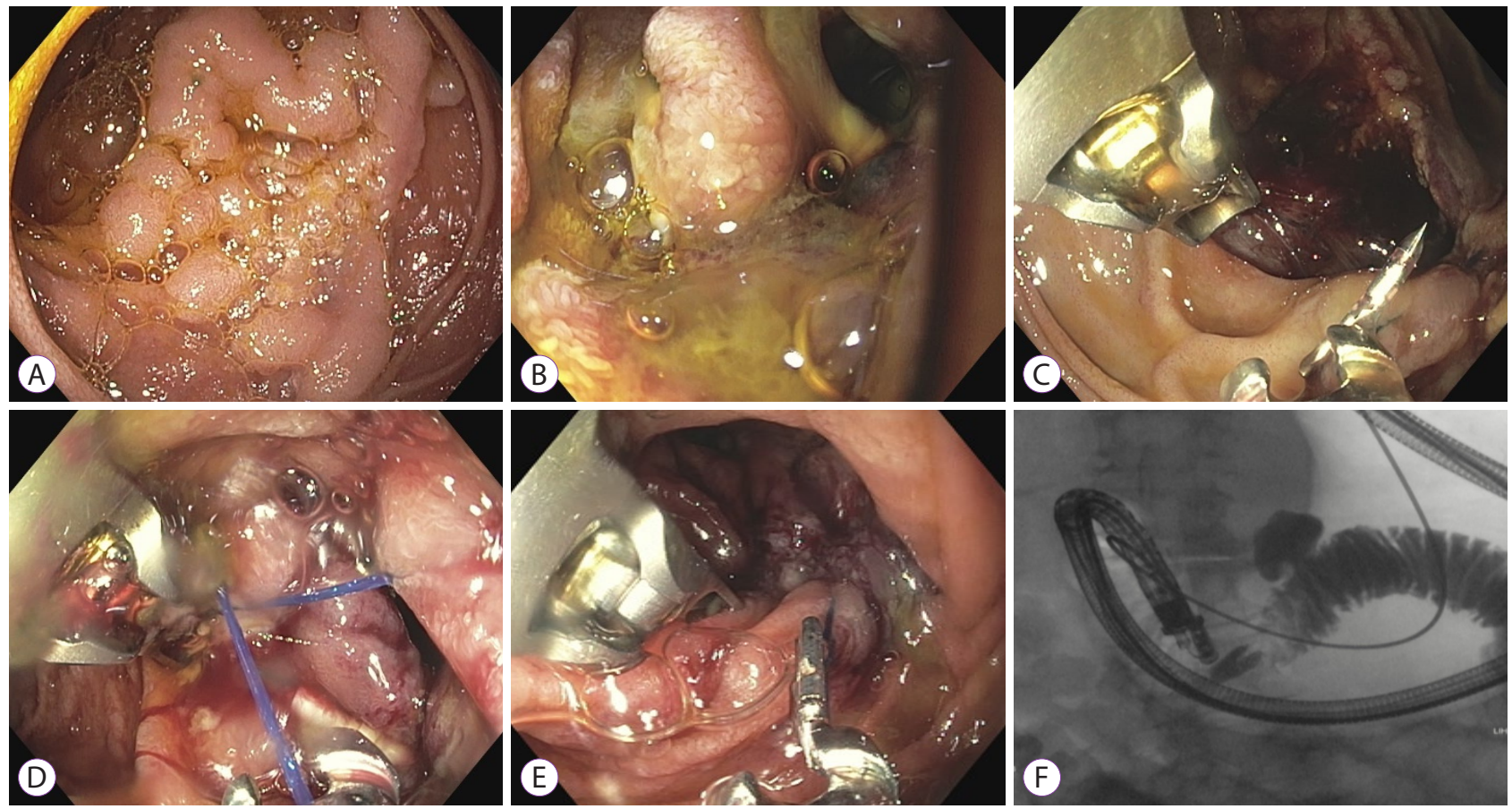

Fig. 1. (A) A 30-mm duodenal adenoma located in the junction of the second and third duodenum. (B) After duodenal endoscopic mucosal resection, prophylactic OverStitch endoscopic suturing was performed. (C) One of the three sutures was loose, and duodenal perforation was observed 5 hours later. (D) The second OverStitch endoscopic suturing was performed. (E) The perforation site was closed using three separate OverStitch endoscopic stitches. (F) Fluoroscopy showed no dye leakage through the duodenum.

plicated ES were discharged immediately after the procedure. Adverse events were noted in two patients. A single episode of postprocedural pancreatitis was noted that required 1 day of hospitalization for observation. Overall, no early or delayed
post-EMR bleeding was recorded. In addition, no clinically obvious duodenal stricture or recurrence was encountered on endoscopic follow-up evaluation, and no patients required subsequent surgical intervention. 


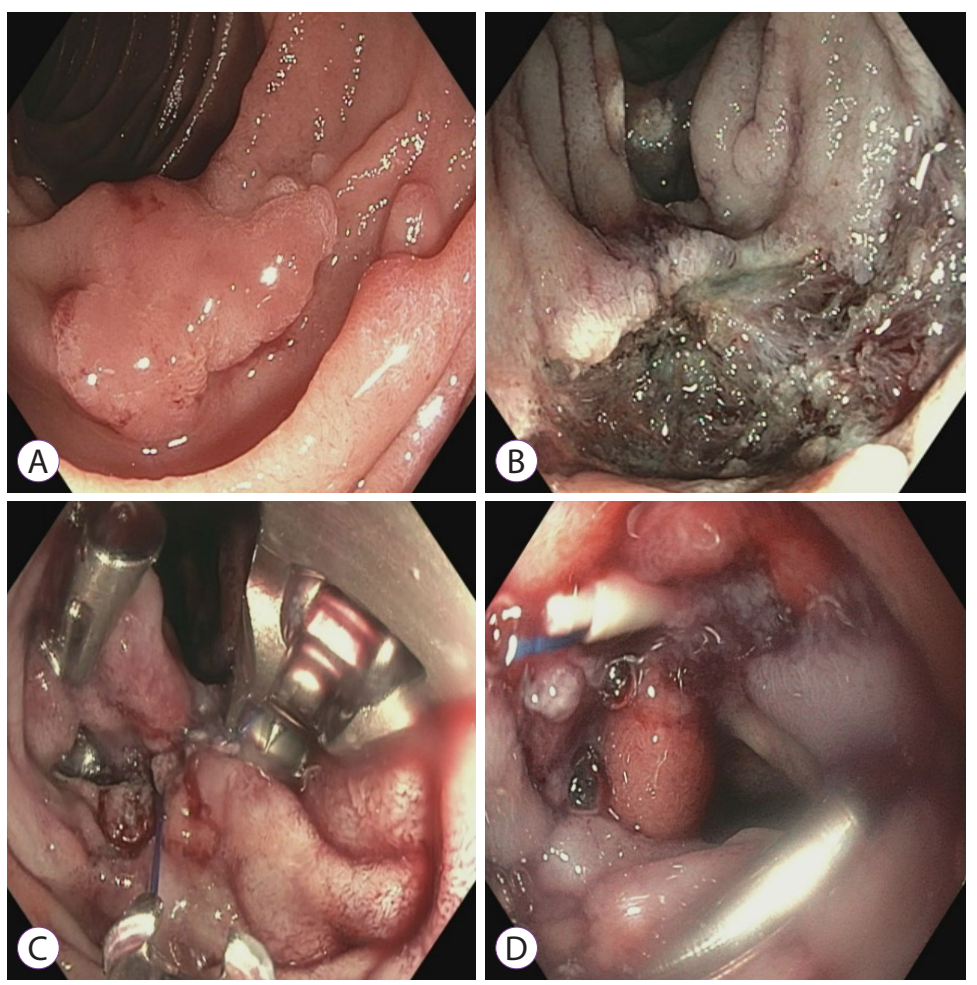

Fig. 2. (A) A 20-mm duodenal adenoma located in the anterior wall of the second part of the duodenum. (B) After duodenal endoscopic mucosal resection. (C) Prophylactic closure was first attempted through endoscopic clip application; however, it failed owing to a large diastasis between the edges and the tangential application. OverStitch endoscopic suturing was then performed with interrupted stitches. (D) The closure of the defect with OverStitch endoscopic suturing.

\section{DISCUSSION}

To our knowledge, this is the first case series focusing on the use of the OverStitch ES device for post-EMR duodenal mucosal defects after the resection of large duodenal mucosal lesions. We demonstrated that prophylactic complete closure of a duodenal mucosal defect is technically feasible. Even duodenal perforations, if discovered early, may be effectively closed with OverStitch ES without the typically difficult and prolonged course. In fact, none of the patients in our small case series required surgical interventions. Our results support the notion that, by apposing the mucosectomy-induced cut edges over a large area together, ES can decrease the risk of delayed perforation. Thus, we have demonstrated an approach for an early and reliable closure of a perforation, which minimizes spillage of food contents and the pro-inflammatory pancreaticobiliary fluid, thereby facilitating recovery and shortening the hospital stay.

Duodenal neoplasms are uncommon, and primary duodenal adenocarcinoma represents only $0.3 \%$ of all malignant neoplasms of the gastrointestinal tract. ${ }^{3,4}$ Approximately 40\% of duodenal adenomas are sporadic, and the remaining 60\% are found in patients with familial adenomatous polyposis., ${ }^{5,6}$ Although there is no definitively established treatment for large duodenal adenomas, EMR is generally considered the first-line treatment for duodenal neoplasms. ${ }^{21,22}$ However, performing duodenal EMR is challenging for multiple reasons. First, the tortuous and narrow lumen poses major difficulty in obtaining a full view of the lesion in a stable position. Second, Brunner's glands in the submucosal layer can stiffen the duodenal wall, making submucosal lifting difficult to achieve. Third, the thinness of the duodenal wall is a predisposing factor for mucosectomy-related perforation. ${ }^{21,22}$ Fourth, exposure of the abundant submucosal duodenal vasculature during EMR increases the risk of postprocedural bleeding. In addition, constant bathing of the postmucosectomy tissue with bile fluid, gastric acid, and pancreatic juice likely predisposes patients to a considerable risk of perforation of the thinned wall.

Delayed perforation has been reported in $1 \%$ of non-ampullary adenomas; however, it may be significantly more frequent after the endoscopic resection of large-sized adenomas. ${ }^{12,13}$ Patients with perforation are typically referred for emergency 
surgery, although it will still result in significant mortality and morbidity. ${ }^{12,14,15}$ The OverStitch ES system is currently the only commercially available, Food and Drug Administration-approved ES device in the United States. ${ }^{23-26}$ It is possible to perform ES to prevent or treat complications associated with duodenal EMR. The literature on the application of ES during duodenal EMR is scarce. Almost all previously described techniques for the closure of post-EMR or post-ESD defects were performed with endoscopic clips, sometimes in combination with endoscopic loops. ${ }^{26-30}$ Clipping, when performed in combination with an endoscopic loop, may be technically challenging or even impossible to accomplish. Besides the obvious difficulty in pulling together the edges of a large duodenal mucosal defect, standard two-arm clips are challenging to orient and to use for capturing tissue in a tangential manner. When placed parallel to the horizontal folds, they risk the creation of duodenal strictures. Over-the-scope clips are easier to deploy than hemostatic clips, and are highly secure and reliable when the defect is perfectly captured. However, they are only suitable for treating tissues $<1.5 \mathrm{~cm}$ in diameter, and precise deployment is absolutely essential. Additionally, they must be properly oriented to prevent stricture formation. A recent report by Fukuhara et al. proposed a multidisciplinary endoscopic approach for managing duodenal perforations. ${ }^{31}$ The authors used a combination of clips, polyglycolic acid sheets, fibrin glue, and endoscopic nasobiliary and pancreatic duct drainage tube insertion. This method utilizes very complicated maneuvers, and its true efficacy warrants further validation.

The OverStitch suture device used in this study was attached to the tip of a double-lumen upper endoscope. Its mobile, curved arm was used to transfer a short needle that carries a suture through the length of one of the instrument channels. With the opening and closing of this arm, the needle was passed back and forth through a targeted tissue, thereby running the stitches through it. The advantage of using this device for closing a duodenal defect is that capturing one of the edges is an independent action from grasping the opposite edge, which is technically much easier to perform than clip placement. Furthermore, placement of the needle on the tissue does not require the precision necessary for clipping. The thread may land on the tissue farther away from the edge, which is healthier and can more strongly hold it in place. Finally, coaxial stitching is relatively easy to perform, thereby reducing the chance of stricture formation to a minimum.

The main limitations of our study were the retrospective, single-center design and the small number of patients. However, our consecutive enrollment of patients who underwent duodenal endoscopic resection was expected to reduce possible selection bias. Moreover, because all procedures were performed by a single skilled endoscopist who has performed a large volume of ES procedures, generalizing our experience to other centers or providers may not be appropriate. In addition, no suturing was performed beyond the D2-D3 junction, which might have been extremely challenging. Large prospective multicenter studies are needed to confirm our preliminary results.

The size of duodenal lesion resection for which ES can be safely performed remains to be determined. However, given the craniocaudal nature of endoscopic sutures, we believe that this technique is feasible for lesions measuring approximately $5 \mathrm{~cm}$ in length. For the same reason, there should be no limitation on the circumferential size of the lesion.

In conclusion, ES for the prevention and treatment of duodenal perforations after EMR is technically feasible, safe, and effective. ES can be considered an option for the prevention and treatment of perforations associated with EMR of large duodenal adenomas.

Conflicts of Interest

The authors have no potential conflicts of interest.

Funding

None.

Author Contributions

Conceptualization: Simon K. Lo

Data curation: Jaeil Chung, Kelly Wang, Alexander Podboy

Writing-original draft: JC, SKL

Writing-review\&editing: JC, KW, AP, Srinivas Gaddam, SKL

ORCID

Jaeil Chung

Kelly Wang

Alexander Podboy

Srinivas Gaddam

Simon K. Lo

https://orcid.org/0000-0001-8920-1359 https://orcid.org/0000-0002-7803-1005 https://orcid.org/0000-0001-9353-4965 https://orcid.org/0000-0001-6818-6305 https://orcid.org/0000-0002-1263-0400

\section{REFERENCES}

1. Jepsen JM, Persson M, Jakobsen NO, et al. Prospective study of prevalence and endoscopic and histopathologic characteristics of duodenal polyps in patients submitted to upper endoscopy. Scand J Gastroenterol 1994;29:483-487.

2. Culver EL, McIntyre AS. Sporadic duodenal polyps: classification, investigation, and management. Endoscopy 2011;43:144-155.

3. Moss WM, McCart PM, Juler G, Miller DR. Primary adenocarcinoma of the duodenum. Arch Surg 1974;108:805-807.

4. Spira IA, Ghazi A, Wolff WI. Primary adenocarcinoma of the duodenum. Cancer 1977;39:1721-1726.

5. Serrano PE, Grant RC, Berk TC, et al. Progression and management of duodenal neoplasia in familial adenomatous polyposis: a cohort study. Ann Surg 2015;261:1138-1144.

6. Groves CJ, Saunders BP, Spigelman AD, Phillips RK. Duodenal cancer in patients with familial adenomatous polyposis (FAP): results of a 10 year prospective study. Gut 2002;50:636-641. 
7. Navaneethan U, Hasan MK, Lourdusamy V, Zhu X, Hawes RH, Varadarajulu S. Efficacy and safety of endoscopic mucosal resection of non-ampullary duodenal polyps: a systematic review. Endosc Int Open 2016;4:E699-E708.

8. Pérez-Cuadrado-Robles E, Quénéhervé L, Margos W, et al. ESD versus EMR in non-ampullary superficial duodenal tumors: a systematic review and meta-analysis. Endosc Int Open 2018;6:E998-E1007.

9. Park SM, Ham JH, Kim BW, et al. Feasibility of endoscopic resection for sessile nonampullary duodenal tumors: a multicenter retrospective study. Gastroenterol Res Pract 2015;2015:692492.

10. Yamamoto H, Miura Y. Duodenal ESD: conquering difficulties. Gastrointest Endosc Clin N Am 2014;24:235-244.

11. Podboy A, Friedland S. Endoscopic submucosal dissection in the West. Int J of Gastrointest Interv 2019;8:106-109.

12. Nonaka S, Oda I, Tada K, et al. Clinical outcome of endoscopic resection for nonampullary duodenal tumors. Endoscopy 2015;47:129-135.

13. Inoue $\mathrm{T}$, Uedo $\mathrm{N}$, Yamashina $\mathrm{T}$, et al. Delayed perforation: a hazardous complication of endoscopic resection for non-ampullary duodenal neoplasm. Dig Endosc 2014;26:220-227.

14. Fanning SB, Bourke MJ, Williams SJ, Chung A, Kariyawasam VC. Giant laterally spreading tumors of the duodenum: endoscopic resection outcomes, limitations, and caveats. Gastrointest Endosc 2012;75:805-812.

15. Matsuda Y, Sakamoto K, Kataoka N, Yamaguchi T, Tomita M, Makimoto $\mathrm{S}$. Perforation associated with endoscopic submucosal dissection for duodenal neoplasm without a papillary portion. World J Gastrointest Surg 2017;9:161-166.

16. Fujihara S, Mori H, Kobara H, et al. Management of a large mucosal defect after duodenal endoscopic resection. World J Gastroenterol 2016;22:6595-6609.

17. Armengol-Miro JR, Dot J, Abu-Suboh Abadia M, et al. New endoscopic suturing device for closure of chronic gastrocutaneous fistula in an immunocompromised patient. Endoscopy 2011;43(Suppl 2):E403-E404.

18. Kantsevoy SV, Bitner M. Esophageal stent fixation with endoscopic suturing device (with video). Gastrointest Endosc 2012;76:1251-1255.

19. Jirapinyo P, Watson RR, Thompson CC. Use of a novel endoscopic suturing device to treat recalcitrant marginal ulceration (with video). Gastrointest Endosc 2012;76:435-439.
20. Bonin EA, Wong Kee Song LM, Gostout ZS, Bingener J, Gostout CJ. Closure of a persistent esophagopleural fistula assisted by a novel endoscopic suturing system. Endoscopy 2012;44(Suppl 2):E8-E9.

21. Alexander S, Bourke MJ, Williams SJ, Bailey A, Co J. EMR of large, sessile, sporadic nonampullary duodenal adenomas: technical aspects and long-term outcome (with videos). Gastrointest Endosc 2009;69:66-73.

22. Honda T, Yamamoto H, Osawa H, et al. Endoscopic submucosal dissection for superficial duodenal neoplasms. Dig Endosc 2009;21:270-274.

23. Kantsevoy SV, Bitner M, Mitrakov AA, Thuluvath PJ. Endoscopic suturing closure of large mucosal defects after endoscopic submucosal dissection is technically feasible, fast, and eliminates the need for hospitalization (with videos). Gastrointest Endosc 2014;79:503-507.

24. Zorron R, Veltzke-Schlieker W, Adler A, et al. Endoscopic sleeve gastroplasty using Apollo Overstitch as a bridging procedure for superobese and high risk patients. Endoscopy 2018;50:81-83.

25. Otake Y, Saito Y, Sakamoto T, et al. New closure technique for large mucosal defects after endoscopic submucosal dissection of colorectal tumors (with video). Gastrointest Endosc 2012;75:663-667.

26. Kantsevoy SV, Thuluvath PJ. Successful closure of a chronic refractory gastrocutaneous fistula with a new endoscopic suturing device (with video). Gastrointest Endosc 2012;75:688-690.

27. Liaquat H, Rohn E, Rex DK. Prophylactic clip closure reduced the risk of delayed postpolypectomy hemorrhage: experience in 277 clipped large sessile or flat colorectal lesions and 247 control lesions. Gastrointest Endosc 2013;77:401-407.

28. Matsuda T, Fujii T, Emura F, et al. Complete closure of a large defect after EMR of a lateral spreading colorectal tumor when using a two-channel colonoscope. Gastrointest Endosc 2004;60:836-838.

29. Sakamoto N, Beppu K, Matsumoto K, et al. "Loop Clip", a new closure device for large mucosal defects after EMR and ESD. Endoscopy 2008;40(Suppl 2):E97-E98.

30. Fujii T, Ono A, Fu KI. A novel endoscopic suturing technique using a specially designed so-called "8-ring" in combination with resolution clips (with videos). Gastrointest Endosc 2007;66:1215-1220.

31. Fukuhara S, Kato M, Iwasaki E, et al. Management of perforation related to endoscopic submucosal dissection for superficial duodenal epithelial tumors. Gastrointest Endosc 2020;91:1129-1137. 